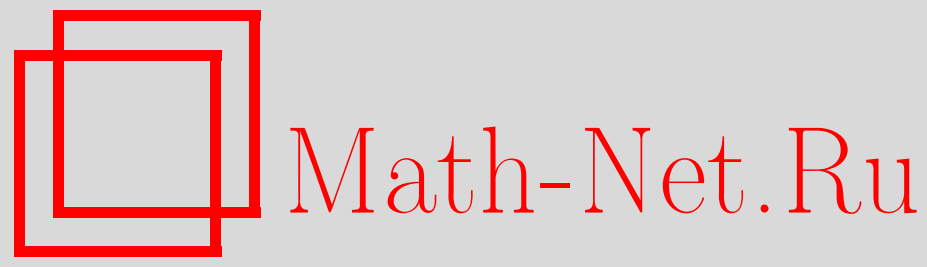

Р. С. Исмагилов, А. Г. Костюченко, О возмущении спектра дифференциального оператора, порожденном ограниченным изменением потенциала, Функи. анализ и его прил., 2009, том 43, выпуск $3,54-64$

DOI: https://doi.org/10.4213/faa2965

Использование Общероссийского математического портала MathNet.Ru подразумевает, что вы прочитали и согласны с пользовательским соглашением

http://www.mathnet.ru/rus/agreement

Параметры загрузки:

IP : 54.81 .137 .203

26 апреля 2023 г., 16:11:11

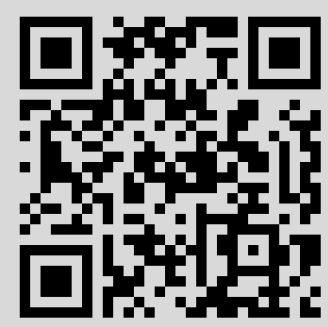


Функиионалъный анализ и его приложения

2009, т. 43, вып. 3, с. 54-64

УДК 517.98

\title{
О возмущении спектра дифференциального оператора, порожденном ограниченным изменением потенциала*
}

\author{
(c) 2009. Р. С. ИСмаГИЛов, А. Г. КОСТЮчЕНКо
}

Памяти Соломона Григорвевича Михлина

\begin{abstract}
Рассматривается возмущение потенциала дифференциального оператора ограниченным измеримым добавочным слагаемым. Доказывается, что если некоторое среднее этого слагаемого имеет предел, то последовательность чезаровских средних возмущения спектра имеет тот же предел.
\end{abstract}

\section{§1. Введение}

Рассматривается симметрический оператор $A$ в $L_{2}\left(\mathbb{R}^{n}\right)$, заданный выражением $P(i \partial / \partial x)+q(x)$, где $P$ - однородный многочлен степени $2 m, P(x)>0$ при $x \neq 0$, функция $q$ гладкая положительная и $q(x) \rightarrow+\infty$ при $|x| \rightarrow \infty$. При этих условиях оператор $A$ самосопряжен и имеет дискретный спектр $\lambda_{1} \leqslant \lambda_{2} \leqslant \ldots$. Возьмем, далее, вещественную измеримую ограниченную функцию $b(x), x \in$ $\mathbb{R}^{n}$, и оператор $\tilde{A}$, полученный из $A$ заменой функции $q$ на $q+b$. Рассмотрим его спектр $\tilde{\lambda}_{1} \leqslant \tilde{\lambda}_{2} \leqslant \ldots$ и величины $\Delta_{k}=\tilde{\lambda}_{k}-\lambda_{k}$ (возмущение спектра). Так как мы не накладываем никаких условий гладкости на функцию $b$, то нельзя ожидать регулярного поведения последовательности $\left\{\Delta_{k}\right\}$. Если, однако, некоторое усреднение функции $b$ имеет предел при $|x| \rightarrow \infty$, то можно надеяться, что и некоторое усреднение последовательности $\Delta_{k}$ имеет предел при $n \rightarrow \infty$ и, вероятно, тот же. Было бы интересно проследить связь между этими двумя средними (одно - в пространстве ограниченных измеримых функций, другое в пространстве числовых последовательностей, стремящихся к бесконечности).

В данной работе (теоремы 2, 3 ниже) используются два способа усреднить возмущение потенциала - оба они являются вариантами усреднения по Риссу; доказывается, что в обоих случаях соответствующее возмущение спектра описывается при помощи чезаровских средних в пространстве ограниченных последовательностей (предела последовательности средних арифметических). В теореме 4 рассматривается возмущение потенциала, для которого риссовское среднее может стремиться к нулю; в этом случае дается более детальное описание возмущения спектра. Эти результаты выводятся из «абстрактной» теоремы о возмущениях (теорема 1).

Для доказательства теорем 2-4 нам понадобится функция Грина параболического уравнения (ядро интегрального оператора $\exp (-t A)$ ), обозначаемая через $G(x, y, t)$, и ее асимптотика при $t \rightarrow+0$, равномерная по $x, y \in \mathbb{R}^{n}$. Эта асимптотика была выведена вторым автором в лекциях [1] сравнительно слож-

*Работа поддержана грантом РФФИ 08-01-00595 и грантом НШ 2372.2008.1. 
ным способом. Мы приведем другой (достаточно короткий) вывод (теорема 5); полученная асимптотическая формула имеет несколько иную форму, чем в [1].

Мы ограничиваемся случаем однородного многочлена $P$, задающего дифференциальный оператор; однако результаты переносятся на более общий случай.

\section{§2. Формулировки теорем}

Начнем с «абстрактной» теоремы о возмущении спектра. Пусть $H$ - гильбертово пространство и $A$ - самосопряженный оператор с дискретным спектром $\lambda_{1} \leqslant \lambda_{2} \leqslant \ldots$. Пусть, далее, $B$ - ограниченный самосопряженный оператор и $\tilde{\lambda}_{1} \leqslant \tilde{\lambda}_{2} \leqslant \ldots-$ спектр оператора $A+B$. Рассмотрим величины $\Delta_{k}=\tilde{\lambda}_{k}-\lambda_{k}$. Положим $w(t)=\operatorname{Tr}(\exp (-t A))$ (эта функция предполагается конечной).

Теорема 1. Справедливо соотношение

$$
\sum_{k=1}^{\infty} \Delta_{k} \exp \left(-t \lambda_{k}\right)=\operatorname{Tr}(B \exp (-t A))+O(t w(t / 3)) \quad \text { nрu } t \rightarrow+0 .
$$

Сумма, записанная в левой части равенства (1), возникала неоднократно при выводе формул «регуляризованных следов». Обстоятельный обзор этой тематики содержится в [2], где можно найти все необходимые ссылки. Мы отметим лишь работу Подольского [3], ибо теорема 1 уточняет один из результатов этой работы. Уточнение заключается в форме остаточного слагаемого в правой части равенства (1).

Сейчас нам понадобится «считающая функция» $N(\lambda)=\max \left\{k: \lambda_{k} \leqslant \lambda\right\}$; подчиним ее условию

$$
N(\lambda) / N\left(\lambda^{\prime}\right) \rightarrow 1 \quad \text { при } \lambda / \lambda^{\prime} \rightarrow 1, \lambda \rightarrow \infty ;
$$

в интересующих нас случаях это условие можно будет легко проверить при помощи асимптотической формулы для этой функции.

Следствие 1. Пусть $\operatorname{Tr}(B \exp (-t A))=(l+o(1)) \operatorname{Tr}(\exp (-t A))$ nрu $t \rightarrow+0$. Пусть, далее, $t w(t / 3)=o(w(t)), t \rightarrow+0$. Тогда

$$
\frac{1}{N} \sum_{k=1}^{N} \Delta_{k} \rightarrow l \quad \text { npu } N \rightarrow \infty .
$$

Это следствие будет выведено (в §3) из теоремы 1 при помощи тауберовой теоремы Караматы.

Обратимся к дифференциальному оператору $A=P(i \partial / \partial x)+q(x)$. Положим $\alpha=2 m /(2 m-1), \beta=1 /(2 m-1), \theta=n /(2 m)$. Наложим на функцию $q$ следующие ограничения.

Существуют такие положительные числа $L, \delta<1+1 /(2 m)$ и $\mu$, что

(i) $|q(x)-q(y)| \leqslant L|x-y| q^{\delta}(x)$ при $|x-y| \leqslant 1$;

(ii) $q(x) / q(y) \leqslant L$ при $|x-y| \leqslant 1$;

(iii) $q(y) \leqslant L q^{\mu}(x) \exp \left(L|x-y|^{\alpha}\right)$ для всех $x, y$;

(iv) функция $v(t)=\int_{\mathbb{R}^{n}} \exp (-t q(x)) d x, t>0$, конечна, и $v(a t) t^{\epsilon}=o(v(t))$ при $t \rightarrow+0$ для некоторого (а потому и для любого) $a \in(0,1)$, где $\epsilon=1+1 /(2 m)-\delta$.

(v) $\int_{\mathbb{R}^{n}} q^{-r}(x) d x<\infty$ для некоторого $r>0$.

Условимся считать (это, разумеется, допустимо), что $\delta \in[1 /(2 m), 1+1 /(2 m))$ и $\mu \geqslant 1$; таким образом, $0<\epsilon \leqslant 1$. 
При доказательстве теорем 2-4 мы опираемся на теорему 5, доказываемую в $\S 5$. В следующей теореме 2 мы усредняем возмущения потенциала по областям вида $\left\{x \in \mathbb{R}^{n}: q(x) \leqslant R\right\}$. Введем функцию $M(v), v \geqslant 0$, и ее среднее порядка $k$ равенствами

$$
M(v)=\int_{q(x)<v} b(x) d x, \quad M_{k}(v)=\frac{1}{(k-1) !} \int_{0}^{v} M(u)(v-u)^{k-1} d u .
$$

Аналогичные функции, построенные заменой функции $b$ на 1 , обозначим через $M^{\circ}$ и $M_{k}^{\circ}$.

Теорема 2. Пусть

$$
M_{k}(v) / M_{k}^{\circ}(v) \rightarrow l \quad n p u \quad v \rightarrow \infty
$$

для некоторого $k \in\{0,1, \ldots\}$. Тогда

$$
\frac{1}{N} \sum_{i=1}^{N} \Delta_{i} \rightarrow l \quad \text { nрu } N \rightarrow \infty .
$$

Для формулировки теоремы 3 понадобится иной вариант усреднения функции $b$. Предположим, что она гладкая (класса $C^{1}$ ). Рассмотрим векторное поле $\nu$ на $\mathbb{R}^{n}$, заданное вне некоторого компакта $K$. Предположим, что для любой точки $x \notin K$ имеется интегральная кривая, которая начинается в точке из $K$, проходит через $x$ и уходит затем в бесконечность. Пусть дана 1 -форма $\omega^{1}$, определенная вне $K$ и удовлетворяющая условиям $\left\langle\omega^{1}, \nu\right\rangle=1, \nu q \geqslant 0$ (здесь взята производная функции $q$ вдоль векторного поля $\nu$ ). (Таким образом, функция $q$ возрастает, если двигаться по интегральной кривой в бесконечность.) Часть этой интегральной кривой от точки, лежащей в $K$, до $x$ обозначим через $s_{x}$. Определим усреднение функции $b$ равенством

$$
b^{\circ}(x)=\int_{s_{x}} b \omega^{1} / \int_{s_{x}} \omega^{1} .
$$

Теорема 3. Пусть

$$
b^{\circ}(x) \rightarrow l \quad \text { nрu }|x| \rightarrow \infty .
$$

Тогда выполняется соотношение (4).

Вернемся к теореме 1 , считая, что $l=0$. В этом случае хотелось бы получить более детальную характеристику возмущения спектра $\left\{\Delta_{k}\right\}$. В следующей теореме мы сделаем это, ограничившись случаем, когда функции $N(\lambda)$ и $M(v)$ имеют степенную асимптотику при $\lambda \rightarrow+\infty$ и $v \rightarrow+\infty$ соответственно. В теореме используется величина $\gamma$, указанная после формулировки теоремы 5 .

Теорема 4. Пусть $N(\lambda) \simeq N_{0} \lambda^{a}$ nрu $\lambda \rightarrow+\infty u M(v) \simeq M_{0} v^{h} n p u v \rightarrow+\infty$, причем числа $M_{0}, N_{0}, a, h$ положстельны и $a>\theta, a-\theta-1<h<a-\theta$. Пусть, далее, функиия $b(x)$ сохраняет постоянныи знак. Тогда

$$
\sum_{k=0}^{n} \Delta_{k} \simeq M_{1} n^{\tau}, \quad n \rightarrow+\infty,
$$

где $\tau=(\theta+h) / a, M_{1}=\gamma M_{0} N_{0}^{-\tau} \Gamma(h+1) / \Gamma(\theta+h+1)$. 
Как уже сказано во введении, доказательство теорем 2-4 использует асимптотику функции Грина при $t \rightarrow+0$. Эта асимптотика описывается в следующей теореме 5. Введем функцию

$$
G_{0}(x, t)=\int_{\mathbb{R}^{n}} \exp (-t P(s)-i(x, s)) d s, \quad x, y \in \mathbb{R}^{n}, t>0 ;
$$

функция Грина оператора $P(i \partial / \partial x)$ (точнее, соответствующего параболического уравнения) выражается через нее как $G_{0}(x-y, t)$. Мы используем величины $\alpha, \beta, \theta, \epsilon$, введенные ранее в этом параграфе.

Теорема 5. Пусть выполнены указанные выше ограничения (i)-(v). Тогда для любого $N$ справедливо соотношение

$$
\begin{aligned}
G(x, y, t)=\exp (-t q(x)) G_{0}(x-y, t) & \\
& +O(1) t^{-\theta+\epsilon} \exp \left(-c\left(t q(x)+\frac{|x-y|^{\alpha}}{t^{\beta}}\right)\right)+O(1) t^{N} q^{-N}(x),
\end{aligned}
$$

где число $c>0$ зависит от $N$, а $O(1)$ означает функцию, ограниченную при $x, y \in \mathbb{R}^{n} u t>0$.

Добавим, что справедливо соотношение $G_{0}(x, x, t)=\gamma t^{-\theta}$, где число $\gamma$ определяется многочленом $P(x)$.

\section{§3. Доказательство теоремы 1 и следствия 1}

Ясно, что $\exp \left(-t \tilde{\lambda}_{k}\right)=\exp \left(-t \lambda_{k}\right)\left(1-t \Delta_{k}+\varepsilon_{k}(t)\right)$, где $\varepsilon_{k}(t)=O\left(t^{2}\right)$ равномерно по $k \in\{1,2, \ldots\}$. Отсюда следует, что

$$
\sum_{k=1}^{\infty} \Delta_{k} \exp \left(-t \lambda_{k}\right)=t^{-1} \operatorname{Tr}(\exp (-t A)-\exp (-t \tilde{A}))+O(t) w(t), \quad t \rightarrow+0 .
$$

Исследуем написанный здесь операторный след. Имеем равенство

$$
\exp (-t A)-\exp (-t \tilde{A})=\int_{0}^{t} \exp (-(t-s) A) B \exp (-s \tilde{A}) d s
$$

(оно проверяется дифференцированием по $t$ и многократно использовалось в литературе). Из него получаем (двукратным применением выписанного здесь интегрального преобразования), что

$$
\begin{aligned}
\exp (-t A)-\exp (-t \tilde{A})=\int_{0}^{t} \exp (-(t-s) A) B \exp (-s A) d s \\
\left.\quad-\int_{D} \exp (-(t-s) A) B \exp \left(-\left(s-s_{1}\right) A\right) B \exp \left(-s_{1} \tilde{A}\right)\right) d s d s_{1},
\end{aligned}
$$

где последнее слагаемое есть интеграл по треугольнику $D=\left\{\left(s_{1}, s\right): 0 \leqslant s_{1} \leqslant\right.$ $s \leqslant t\}$. Оценим ядерную норму этого слагаемого. Для этого используем известное неравенство для ядерной нормы: $\left\|T_{1} T_{2} T_{3}\right\|_{1} \leqslant\left\|T_{1}\right\|\left\|T_{2}\right\|_{1}\left\|T_{3}\right\|$ (см. [5, с. 122]). Величины $t-s, s-s_{1}$ и $s_{1}$, участвующие в нашем интеграле, неотрицательны, и их сумма равна $t$. Следовательно, хотя бы одна из них не меньше величины $t / 3$; пусть, например, $s-s_{1} \geqslant t / 3$. Тогда $\left\|\exp \left(-\left(s-s_{1}\right) A\right)\right\|_{1} \leqslant w(t / 3)$, и из указанного неравенства следует, что ядерная норма подынтегральной функции не превосходит величины $\|B\|^{2} w(t / 3)$, а потому ядерная норма последнего 
слагаемого в (9) не превосходит величины $\|B\|^{2} w(t / 3) t^{2}$. Аналогично оценивается и ядерная норма первого интеграла в (9); его след вычисляется очевидным образом и равен $t \operatorname{Tr}(\exp (-t A) B)$. Получаем, что

$$
\operatorname{Tr}(\exp (-t \tilde{A})-\exp (-t A))=t \operatorname{Tr}(\exp (-t A) B)+w(t / 3) O\left(t^{2}\right) .
$$

Отсюда и из (8) получаем теорему 1.

Докажем следствие 1. Для этого применим тауберову теорему Караматы [6]; напомним ее. Пусть $A(\lambda), \lambda \geqslant 0$, - неубывающая неограниченная функция, удовлетворяющая условиям $A(0) \geqslant 0, A(\lambda) / A\left(\lambda^{\prime}\right) \rightarrow 1$ при $\lambda / \lambda^{\prime} \rightarrow 1, \lambda \rightarrow \infty$, a функция $s(\lambda), \lambda \geqslant 0$, непрерывна и ограничена снизу. Пусть

$$
\int_{0}^{\infty} \exp (-t \lambda) s(\lambda) d A(\lambda)=(l+o(1)) \int_{0}^{\infty} \exp (-t \lambda) d A(\lambda) \quad \text { при } t \rightarrow+0 .
$$

Тогда

$$
\frac{1}{A(x)} \int_{0}^{x} s(\lambda) d A(\lambda) \rightarrow l \quad \text { при } x \rightarrow+\infty .
$$

Чтобы применить теорему Караматы к следствию 1 , возьмем в качестве $A(\lambda)$ считающую функцию $N(\lambda)$, а в качестве $s(\lambda)$ - кусочно-линейную функцию, удовлетворяющую условию $s\left(\lambda_{k}\right)=\Delta_{k}$. Напомним, что выполнено также условие (2), которое требуется в теореме Караматы. Отсюда вытекает следствие 1.

\section{§4. Доказательство теорем 2, 3, 4}

Обратимся к дифференциальному оператору $A=P(i \partial / \partial x)+q(x)$ и докажем теоремы 2 и 3, используя следствие 1 и теорему 5 (последняя доказывается в $\S 5)$. Возьмем в качестве $B$ оператор умножения на функцию $b(x)$. Мы пользуемся обозначением $w(t)=\operatorname{Tr}(\exp (-t A))$, введенным в $\S 2$. Из формулы $(7)$, записанной при $x=y$, получается асимптотика для функции $G(x, x, t)$, равномерная при $x \in \mathbb{R}^{n}$. Из нее следует (здесь мы используем условие (v) из $\S 2$ ), что

$$
\operatorname{Tr}(B \exp (-t A))=\gamma t^{-\theta} \int_{\mathbb{R}^{n}} b(x) \exp (-t q(x)) d x+o(w(t)), \quad t \rightarrow+0 .
$$

В частности, взяв функцию $b(x)=1$, получаем асимптотическую формулу для $w(t)$ при $t \rightarrow+0$. Применим к операторам $A$ и $B$ следствие 1 ; согласно условию (iv) и неравенству $0<\epsilon \leqslant 1$, предпосылки этого следствия выполнены. Оно сводит доказательство теорем 2 и 3 к проверке соотношения

$$
\int_{\mathbb{R}^{n}} b(x) \exp (-t q(x)) d x=(l+o(1)) \int_{\mathbb{R}^{n}} \exp (-t q(x)) d x .
$$

Начнем с теоремы 2. Для любой суммируемой функции $\phi(u), u \in \mathbb{R}$, справедливо равенство

$$
\int_{\mathbb{R}^{n}} b(x) \phi(q(x)) d x=\int_{0}^{\infty} \phi(u) d M(u) .
$$

(Вот простейший способ способ доказать это равенство. Достаточно рассмотреть случай, когда функция $b$ положительна и интеграл от нее по $\mathbb{R}^{n}$ равен единице; таким образом, возникает вероятностная мера на $\mathbb{R}^{n}$. Тогда доказываемое равенство есть выражение математического ожидания случайной величины через ее функцию распределения.) Запишем это равенство для функции 
$\phi(u)=\exp (-t u)$. Интегрирование по частям приводит правую часть к виду $t^{k+1} \int_{0}^{\infty} M_{k}(u) \exp (-t u) d u$. Аналогично

$$
\int_{\mathbb{R}^{n}} \exp (-t q(x)) d x=t^{k+1} \int_{0}^{\infty} M_{k}^{\circ}(u) \exp (-t u) d u .
$$

По условию теоремы $1 M_{k}(v)=(l+o(1)) M_{k}^{\circ}(v)$ при $v \rightarrow+\infty$, откуда следует (10). Этим доказана теорема 2.

Докажем теорему 3. Положим $k(x)=\int_{s_{x}} \omega^{1}$. Тогда $b=\nu\left(b^{\circ} r\right)$, откуда

$$
\begin{aligned}
\int_{\mathbb{R}^{n}} b(x) \exp (-t q(x)) d x & =-\int_{\mathbb{R}^{n}} \nu\left(\exp (-t q(x)) b^{\circ}(x) r(x)\right) d x \\
& =t \int_{\mathbb{R}^{n}} b^{\circ}(x) r(x) \nu q(x) \exp (-t q(x)) d x .
\end{aligned}
$$

Пользуясь условиями теоремы 3 , заменим в последнем интеграле $b^{\circ}$ на $l+o(1)$ и проведем данную выкладку в обратном порядке (при этом используется условие $\nu q \geqslant 0)$. В итоге приходим к соотношению (10); отсюда следует теорема 3.

Докажем теорему 4. Легкий подсчет (с использованием теоремы 5 и условий теоремы 4) приводит к соотношению

$$
\operatorname{Tr}(B \exp (-t A)) \simeq M_{0} \gamma \Gamma(h+1) t^{-\theta-h}, \quad t \rightarrow+0 .
$$

С другой стороны, при $c \in(0,1)$ справедливо соотношение

$$
\sum_{k=0}^{\infty} k^{-c} \exp \left(-t \lambda_{k}\right) \simeq N_{0}^{1-c} a \Gamma(a-a c) t^{a c-a}, \quad t \rightarrow+0 .
$$

Применим его, взяв $c=1-(\theta+h) a^{-1}$; при этом выполняется равенство $\theta+h=$ $a-a c$. В итоге получаем

$$
\sum_{k=0}^{\infty} \Delta_{k} \exp \left(-t \lambda_{k}\right) \simeq R \sum_{k=0}^{\infty} k^{-c} \exp \left(-t \lambda_{k}\right), \quad t \rightarrow+0,
$$

где $R=M_{0} N_{0}^{-\tau} \gamma \Gamma(h+1)(\Gamma(h+\theta))^{-1} a^{-1}$. Применим уже приведенную тауберову теорему, взяв в качестве функции $A(\lambda)$ функцию со скачками $k^{-c}$ в точках $\lambda_{k}$. Будем считать (для определенности), что функция $b$ неотрицательна; таким образом, $\Delta_{k} \geqslant 0$, чем обеспечено условие тауберовой теоремы. Это дает теорему 4 .

\section{§5. Доказательство теоремы 5}

Разобьем доказательство на шаги.

5а. Интегральное уравнение для $\boldsymbol{G}(\boldsymbol{x}, \boldsymbol{y}, \boldsymbol{t})$. Функция $G(x, y, t)$ есть решение задачи Коши

$$
G_{t}^{\prime}=(-P(i \partial / \partial x)-q(x)) G, \quad G(x, 0)=\delta(x-y) .
$$

Применим к ней обычный метод (использование параметрикса). Возьмем точку $x_{0}$, перепишем уравнение в виде

$$
G_{t}^{\prime}=\left(-P(i \partial / \partial x)-q\left(x_{0}\right)\right) G+\left(q\left(x_{0}\right)-q(x)\right) G
$$


и, считая (временно) последнее слагаемое известным, решим это уравнение; решение выражается через функцию Грина $\exp \left(-t q\left(x_{0}\right)\right) G_{0}(x-y, t)$ для оператора $-P(i \partial / \partial x)-q\left(x_{0}\right)$. Получаем интегральное уравнение для функции $G$. Заменим в нем $x_{0}$ на $x$. В итоге получаем следующее интегральное уравнение:

$$
G=K_{0} G+f_{0}
$$

Здесь $K_{0}$ - интегральный оператор, действующий по формуле

$$
\left(K_{0} f\right)(x, y, t)=\int_{\mathbb{R}^{n} \times[0, t]} K(x, \xi ; t, s) f(\xi, y, s) d \xi d s,
$$

где

$$
K(x, \xi ; t, s)=\exp (-(t-s) q(x)) G_{0}(x-\xi, t-s)(q(x)-q(\xi)),
$$

а $f_{0}(x, y, t)=\exp (-t q(x)) G_{0}(x-y, t)$. В качестве области определения оператора $K_{0}$ возьмем множество всех непрерывных функций $f$, для которых написанный интеграл, выражающий $K_{0} f$, сходится абсолютно (то, что эта область может быть не инвариантной относительно оператора, несущественно). Решение уравнения выражается (пока формально) в виде ряда $f_{0}+K_{0} f_{0}+\ldots$ Наша цель показать, что ряд сходится к искомой функции.

5b. Оценка ядра $\boldsymbol{K}$ и функции $\boldsymbol{f}_{\mathbf{0}}$. Будем пользоваться величинами $\alpha$, $\beta, \theta, \epsilon$, введенными в $\S 2$. Используются всевозможные постоянные $a_{i}, b_{i}$ и пр., нередко без пояснений; будем свободно увеличивать либо уменьшать эти постоянные, а также использовать одну букву в разных оценках.

Известно, что

$$
\left|G_{0}(x, t)\right| \leqslant a_{0} t^{-\theta} \exp \left(-a|x|^{\alpha} t^{-\beta}\right)
$$

$\left(a, a_{0}\right.$ выражаются через $P$; если необходимо, мы будем заменять число $a_{0}$ большим, а $a$ - меньшим числом.) Это неравенство выводится стандартным методом оценки преобразований Фурье (см. [4, с. 35]). Отсюда получаются следующие оценки ядра $K$ и функции $f_{0}$ (в этих оценках указанное только что число а заменено на $\min \{1, a\})$ :

$$
\begin{aligned}
|K(x, \xi ; t, s)| \leqslant & a_{0}(t-s)^{-\theta} \exp \left(-a\left((t-s) q(x)+\frac{|x-\xi|^{\alpha}}{(t-s)^{\beta}}\right)\right)|q(x)-q(\xi)|, \\
& \left|f_{0}(x, y, t)\right| \leqslant a_{0} t^{-\theta} \exp \left(-a\left(t q(x)+\frac{|x-y|^{\alpha}}{t^{\beta}}\right)\right) .
\end{aligned}
$$

5с. Пространства $\boldsymbol{F}_{\boldsymbol{\sigma}}$ и основные леммы. Начнем с простой технической леммы; в ней использовано обозначение $r^{-}=\min \{r, 0\}$.

Лемма 1. Для любых $a>0, \beta>0, \sigma \neq-1$ справедливо неравенство

$$
\int_{0}^{t} \exp \left(-a / s^{\beta}\right) s^{\sigma} d s \leqslant H \exp \left(-a / t^{\beta}\right) a^{(\sigma+1)^{-} / \beta}\left(a+t^{\beta}\right)^{-1-(\sigma+1)^{-} / \beta} t^{\sigma+\beta+1},
$$

где число $H$ зависит от $\sigma, \beta$ (и не зависит от а $u t)$.

Для доказательства следует сделать замену $a / s^{\beta}=v$ и затем применить очевидные асимптотические соотношения для интеграла $\int_{h}^{\infty} \exp (-v) v^{l} d v$ при $h \rightarrow+\infty$ и при $h \rightarrow+0$.

Перейдем к основным леммам. 
Для каждого числа $\sigma$ обозначим через $F_{\sigma}$ пространство всех непрерывных функций $f(x, y, t), x, y \in \mathbb{R}^{n}, 0<t<1$, со свойством

$$
|f(x, y, t)| \leqslant b_{0} t^{\sigma} \exp \left(-b\left(t q(x)+\frac{|x-y|^{\alpha}}{t^{\beta}}\right)\right)
$$

(для некоторых $b, b_{0}$ ). Из (14) следует, что $f_{0} \in F_{-\theta}$.

Лемма 2. Если $f \in F_{\sigma}, \sigma \geqslant-\theta$, mо $K_{0} f=f_{1}+f_{2}$, әде $f_{1} \in F_{\sigma+\epsilon} u$ $\left|f_{2}(x, y, t)\right| \leqslant R t^{M} q^{-M}(x) ;$ здесь $M$ произвольно, а $R$ зависит от $M$.

Доказательство. Введем область $D=\mathbb{R}^{n} \times[0, t]$ и ее подмножества $D_{1}=$ $\{(\xi, s):|\xi-x| \leqslant 1,0 \leqslant s \leqslant t\}$ и $D_{2}=\{(\xi, s):|\xi-x| \geqslant 1,0 \leqslant s \leqslant t\}$. Нам надлежит оценить функцию

$$
\left(K_{0} f\right)(x, y, t)=\int_{D} K(x, \xi ; t, s) f(\xi, y, s) d \xi d s .
$$

Обозначим через $f_{1}$ и $f_{2}$ функции, полученные как аналогичные интегралы по областям $D_{1}$ и $D_{2}$. Докажем, что эти функции удовлетворяют требованиям, указанным в теореме.

Согласно оценке (13), для этого достаточно оценить интегралы вида

$$
\begin{aligned}
I_{k}=\int_{D_{k}}(t-s)^{-\theta} \mid q(x)-q(\xi) & \mid \exp (-c((t-s) q(x)+s q(\xi) \\
+ & \left.\left.\frac{|x-\xi|^{\alpha}}{(t-s)^{\beta}}+\frac{|y-\xi|^{\alpha}}{s^{\beta}}\right)\right) s^{\sigma} d \xi d s, \quad k=1,2 .
\end{aligned}
$$

(Здесь в качестве числа $c$ можно взять $\min \{a, b\}$.)

Докажем, что

$$
I_{1} \leqslant c_{0} t^{\sigma+\epsilon} \exp \left(-c_{1}\left(t q(x)+\frac{|x-y|^{\alpha}}{t^{\beta}}\right)\right)
$$

и

$$
I_{2} \leqslant c_{0}^{\prime} t^{M} q^{-M}(x)
$$

с некоторыми постоянными $c_{0}, c_{1}, c_{0}^{\prime}$ (число $M$ произвольно, $c_{0}^{\prime}$ зависит от $M$ ). Этим будет доказана лемма 2. Мы будем пользоваться свойствами (i)-(v) функции $q$, приведенными в 2 .

Начнем с $I_{1}$; эта величина задана интегралом (16), где интегрирование производится по $D_{1}$. Оценим выражения, входящие в экспоненты из (16). Из условия (ii) следует, что $(t-s) q(x)+s q(\xi) \geqslant c_{1} t q(x)$. Далее, используя очевидное неравенство $u^{\alpha}+v^{\alpha} \geqslant 2^{1-\alpha}(u+v)^{\alpha}, u \geqslant 0, v \geqslant 0$, заменим $|x-\xi|^{\alpha} /(t-s)^{\beta}+|y-\xi|^{\alpha} / s^{\beta}$ на $\frac{1}{2}\left(|x-\xi|^{\alpha} /(t-s)^{\beta}+|y-\xi|^{\alpha} / s^{\beta}\right)+2^{-\alpha}|x-y|^{\alpha} / t^{\beta}$. Наконец, используем оценку (i) для $|q(x)-q(\xi)|$. В итоге приходим к неравенству

$$
I_{1} \leqslant b_{1} \exp \left(-c_{1}\left(t q(x)+|x-y|^{\alpha} / t^{\beta}\right)\right) q^{\delta}(x) T,
$$

где (здесь переходим к интегрированию по $D=\mathbb{R}^{n} \times[0, t]$ )

$$
T=\int_{D}(t-s)^{-\theta}|x-\xi| s^{\sigma} \exp \left(-c_{2}\left(\frac{|x-\xi|^{\alpha}}{(t-s)^{\beta}}+\frac{|y-\xi|^{\alpha}}{s^{\beta}}\right)\right) d \xi d s .
$$

В последнем выражении выполним сначала интегрирование по $s$. Оценим сверху результат интегрирования, используя лемму 1. Для этого возьмем сначала 
интеграл по $[0, t / 2]$ (при этом можно заменить $t-s$ на $t / 2$ ); это приводит к интегралу вида (15) (в котором $t$ заменено на $t / 2$ ). Затем возьмем интеграл по $[t / 2, t]$ (и заменим $s$ на $t / 2$ ); замена переменной $t-s=u$ снова приводит к интегралу вида (15). В итоге получаем, что $T$ не превосходит суммы следующих величин, возможно, умноженных на постоянную (здесь мы переходим к интегрированию по $\left.\mathbb{R}^{n}\right)$ :

$$
\begin{aligned}
\int_{\mathbb{R}^{n}} \exp \left(-c\left(|x-\xi|^{\alpha}+\right.\right. & \left.\left.|y-\xi|^{\alpha}\right) t^{-\beta}\right) t^{\sigma+\beta+1-\theta} \\
& \times|x-\xi||y-\xi|^{\alpha \beta^{-1}(\sigma+1)^{-}}\left(|y-\xi|^{\alpha}+t^{\beta}\right)^{-1-(\sigma+1)^{-} \beta^{-1}} d \xi
\end{aligned}
$$

и

$$
\begin{aligned}
\int_{\mathbb{R}^{n}} \exp \left(-c\left(|x-\xi|^{\alpha}+\right.\right. & \left.\left.|y-\xi|^{\alpha}\right) t^{-\beta}\right) t^{\sigma+\beta+1-\theta} \\
& \times|x-\xi|^{1+\alpha \beta^{-1}(-\theta+1)^{-}}\left(|x-\xi|^{\alpha}+t^{\beta}\right)^{-1-(-\theta+1)^{-} \beta^{-1}} d \xi .
\end{aligned}
$$

Чтобы оценить первое слагаемое, заменим $|x-\xi| \exp \left(-c|x-\xi|^{\alpha} / t^{\beta}\right)$ большей величиной $d_{1} t^{\beta / \alpha}$, затем в полученном интеграле произведем замену $y-\xi=$ $t^{\beta / \alpha} v$. В итоге получаем $d_{2} t^{\sigma+(n+1) \beta / \alpha+1-\theta}$, что совпадает с $d_{2} t^{\sigma+1+1 /(2 m)}$. Чтобы оценить второе слагаемое, отбросим из подынтегрального выражения множитель $\exp \left(-c|y-\xi|^{a l} / t^{\beta}\right)$ и полученный интеграл преобразуем по описанному образцу (замена $x-\xi=t^{\beta / \alpha} v$ ); это дает тот же итог $d_{2} t^{\sigma+1+1 /(2 m)}$ (возможно, с другой постоянной $\left.d_{2}\right)$. Итак, мы доказали, что $T \leqslant d_{3} t^{\sigma+1+1 /(2 m)}$. Теперь для $I_{1}$ получаем неравенство

$$
I_{1} \leqslant d_{0} \exp \left(-d_{1}\left(t q(x)+|x-y|^{\alpha} / t^{\beta}\right)\right) t^{\sigma+1+1 /(2 m)} q^{\delta}(x) .
$$

Применяя неравенство $q^{\delta}(x) \leqslant d_{3} t^{-\delta} \exp \left(d_{4} t q(x)\right), d_{4}<d_{1}$, получаем утверждение (17).

Обратимся к интегралу $I_{2}$. Здесь мы опишем нужные преобразования, не записывая их явно. Во-первых, заменим $|q(x)-q(\xi)|$ на $L q^{\mu}(x) \exp \left(L|x-\xi|^{\alpha}\right)$ (используя условие (iii)). Далее, отбросим множитель $(t-s)^{-\theta} \exp \left(L|x-\xi|^{\alpha}\right)$, ибо он «подавляется» множителем $\exp \left(-|x-\xi|^{\alpha}(t-s)^{-\beta}\right)($ напомним, что $|x-\xi| \geqslant 1)$. Опустим слагаемое $s q(\xi)$. Наконец, перейдем к интегрированию по всей области $D$. Получаем

$$
I_{2} \leqslant b_{2} \int_{D} \exp \left(-c\left((t-s) q(x)+|x-\xi|^{\alpha}(t-s)^{-\beta}+|y-\xi|^{\alpha} s^{-\beta}\right)\right) s^{\sigma} q^{\mu}(x) d \xi d s .
$$

Далее, используя неравенство

$$
a x+b x^{-\beta}>a^{\beta /(\beta+1)}(b \beta)^{(\beta+1)^{-1}}, \quad a>0, b>0, x>0
$$

(оно получается вычислением минимума его левой части), заменим $(t-s) q(x)+$ $|x-\xi|^{\alpha} /(t-s)^{\beta}$ на $c\left(q^{\beta /(\beta+1)}(x)+1 / t^{\beta}\right)$. В итоге приходим к интегралу по $\xi$, который вычисляется явно и равен $c^{\prime} t^{\sigma-\theta}$. Итак,

$$
I_{2} \leqslant b_{3} q^{\mu}(x) \exp \left(-c\left(q^{\beta /(\beta+1)}(x)+1 / t^{\beta}\right)\right) t^{\sigma} .
$$

Отсюда следует неравенство (18).

Лемма доказана.

Лемма 3. Пусть $|f(x, y, t)| \leqslant t^{M} q^{-N}(x)$ (для некоторых $\left.M, N\right)$. Тогда справедливо неравенство $\left|\left(K_{0} f\right)(x, y, t)\right| \leqslant B t^{M+\epsilon} q^{-N}(x)$. 
Здесь $B$ не зависит от $M$ (но может зависеть от $N$ ).

Доказательство. По аналогии с доказательством леммы 2 введем величины

$$
J_{k}=\int_{D_{k}} \exp \left(-c\left((t-s) q(x)+\frac{|x-\xi|^{\alpha}}{(t-s)^{\beta}}\right)\right)(t-s)^{-\theta}|q(x)-q(\xi)| \frac{s^{M}}{q^{N}(\xi)} d \xi d s,
$$

$k=1,2$. Достаточно проверить, что

$$
J_{k} \leqslant a t^{M+\epsilon} q^{-N}(x),
$$

где $a$ не зависит от $M$. При оценке интеграла $J_{1}$ заменим $|q(x)-q(\xi)|$ на $|x-\xi| q^{\delta}(x)$ (пользуясь неравенством (i)), $q^{N}(\xi)$ на $L^{-N} q^{N}(x)$ (используя условие (ii)) и, наконец, заменяем интегрирование по области $|x-\xi| \leqslant 1$ на интегрирование по $\mathbb{R}^{n}$. После этих замен интеграл по $\xi$ вычисляется явно (заменой $\left.x-\xi=(t-s)^{\beta / \alpha} v\right)$, откуда следует, что

$$
J_{1} \leqslant q^{\delta-N}(x) \int_{0}^{t} \exp (-c((t-s) q(x)))(t-s)^{1 /(2 m)} s^{M} d s .
$$

Заменим $q^{\delta}(x) \exp (-(t-s) q(x))$ большей величиной $b_{4}(t-s)^{-\delta}$ и проинтегрируем по $s$. Это дает $J_{1} \leqslant b_{5} t^{M+\epsilon} q^{-N}(x)$, что и требовалось.

Оценим $J_{2}$. Для этого заменим величину $|q(x)-q(\xi)|$ большей величиной $q^{\mu}(x) \exp \left(L|x-\xi|^{\alpha}\right)$ (использовали условие (iii)), отбросим множители $(t-s)^{-\theta}$, $\exp \left(L|x-\xi|^{\alpha}\right)$ (ибо они «подавляются» множителем $\exp \left(-c|x-\xi|^{\alpha} /(t-s)^{\beta}\right.$ в силу условия $|x-\xi| \geqslant 1)$, а также отбросим $q^{N}(\xi)$. Наконец, используем неравенство (19). В итоге получаем, что $J_{2} \leqslant \exp \left(-c\left(q^{\beta /(\beta+1)}(x)+1 / t^{\beta}\right)\right) t^{M}$. Теперь ясно, что условие $(20)$ выполнено также для $J_{2}$. Лемма 3 доказана.

Теперь докажем теорему 5. С этой целью рассмотрим члены ряда $f_{0}+f_{1}$ $+\ldots, f_{k}=K_{0}^{k} f_{0}$, дающего формальное решение интегрального уравнения (11). В дальнейшем $C_{k}$ и пр. - это некоторые постоянные.

Согласно лемме 2 , функцию $f_{k}$ можно представить в виде $f_{k}=f_{k 1}+f_{k 2}$, где $f_{k 1} \in F_{-\theta+k \epsilon}$, т. е. $\left|f_{k 1}\right| \leqslant C_{k} t^{-\theta+k \epsilon} \exp \left(-a_{k}\left(t q(x)+|x-y|^{\alpha} t^{-\beta}\right)\right)$ с некоторыми положительными $C_{k}, a_{k}$, и $\left|f_{k 2}\right| \leqslant R t^{M} q^{-N}(x)$, где $M$ произвольно, а $R$ зависит от $M$. Зафиксируем число $M_{0}$ и возьмем такой номер $k_{0}$, что $-\theta+k_{0} \epsilon>$ $2 M_{0}$. Тогда $\left|f_{k_{0} 1}\right| \leqslant D t^{2 M_{0}} \exp \left(-c\left(t q(x)+b|x-y|^{\alpha} t^{-\beta}\right)\right) \leqslant D t^{2 M_{0}} \exp (-c t q(x)) \leqslant$ $D t^{M_{0}} q^{-M_{0}}(x)$. Применяя к $f_{k_{0} 1}$ лемму 3 , получаем, что дальнейшие члены ряда удовлетворяют неравенствам $\left|f_{k_{0}+j}\right| \leqslant D_{1} B^{j} t^{M_{0}+j \epsilon} q^{-M_{0}}(x)$. Таким образом, ряд $f_{k_{0}}+f_{k_{0}+1}+\cdots$ мажорируется рядом, сходящимся при $t<B^{1 / \epsilon}$, и его сумма не превосходит величины $D_{1} t^{M_{0}} q^{-M_{0}}(x)$. С другой стороны, $\left|f_{01}+\cdots+f_{k_{0}}\right| \leqslant$ $D t^{-\theta+\epsilon} \exp \left(-a^{\prime}\left(t q(x)+|x-y|^{\alpha} t^{-\beta}\right)\right)$. Это приводит к утверждению теоремы 5.

\section{§6. Заключительные замечания}

1. Приведем пример к теореме 2 с целью показать, что для данной функции $b$ величина предела, указанного в условии (3), зависит от выбора функции $q$. Пусть $n=2, k=0$ и $b$ есть индикатор множества $x_{2} \geqslant\left|x_{1}\right|$. Взяв число $h \in(0,1)$, рассмотрим потенциал $q\left(x_{1}, x_{2}\right)=\max \left\{x_{1}, x_{2} / h\right\}$. Тогда предел в $(3)$ равен $h / 4$ и, таким образом, зависит от выбора потенциала. 
2. Рассмотрим факторпространства $L_{\infty}\left(\mathbb{R}^{n}\right) / L_{\infty}^{0}\left(\mathbb{R}^{n}\right)$ и $l_{\infty} / l_{\infty}^{0}$, где через $L_{\infty}\left(\mathbb{R}^{n}\right)$ и $l_{\infty}^{0}$ обозначены подпространства функций (соответственно последовательностей), сосредоточенных на компакте (соответственно на конечном подмножестве). Отображение $b \mapsto\left\{\Delta_{k}\right\}$, рассмотренное выше, приводит к отображению $L_{\infty}\left(\mathbb{R}^{n}\right) / L_{\infty}^{0}\left(\mathbb{R}^{n}\right) \rightarrow l_{\infty} / l_{\infty}^{0}$. Линейно ли оно? (Мы отчасти изучили это отображение лишь на подпространстве элементов указанного факторпространства, имеющих риссовское среднее некоторого порядка.)

\section{ЛитеРАтУРА}

[1] А. Г. Костюченко, Асимптотическое поведение спектральной функиии самосопряженных эллиптических операторов, в кн.: Четвертая математическая школа, Киев, 1968, 42-117.

[2] В. А. Садовничий, В. Е. Подольский, Следы операторов, УМН, 61:5(371) (2006), 89-356.

[3] В. Е. Подольский, О следах возмущенной операторной полугруппы, Матем. заметки, 75:3 (2004), 462-466.

[4] С. В. Эйдельман, Параболические системъ, Наука, М., 1964.

[5] И. Ц. Гохберг, М. Г. Крейн, Введение в теорию несамосопряженных операторов, Наука, М., 1965.

[6] D. Borwein, Tauberian theory, a century of developments, by Jacob Korevaar, Bull. Amer. Math. Soc., 42:3, 401-406.

Московский государственный технический

Поступило в редакцию университет им. Н. Э. Баумана

17 мая 2009 г.

e-mail: ismagil@ci.net.ru

Московский государственный университет им. М. В. Ломоносова

e-mail: akostyuch@mail.ru 